\title{
Induction of the synthesis of an additional family of long-chain dolichols in the yeast Saccharomyces cerevisiae. Effect of starvation and ageing ${ }^{*}$
}

\author{
Anna Szkopinska ${ }^{\bowtie}$, Ewa Swiezewska and Joanna Rytka \\ Institute of Biochemistry and Biophysics, Polish Academy of Sciences, Warszawa, Poland
}

Received: 16 May, 2002; revised: 12 August, 2002; accepted: 20 August, 2002

Key words: yeast, cis-prenyltransferases, glucose, nitrogen starvation

The yeast Saccharomyces cerevisiae strain W303 synthesizes in the early logarithmic phase of growth dolichols of 14-18 isoprene residues. The analysis of the polyisoprenoids present in the stationary phase revealed an additional family which proved to be also dolichols but of 19-24 isoprene residues, constituting $39 \%$ of the total dolichols. The transfer of early logarithmic phase cells to a starvation medium lacking glucose or nitrogen resulted in the synthesis of the longer chain dolichols. The additional family of dolichols represented $\mathbf{1 3 . 8 \%}$ and $\mathbf{1 0 . 3 \%}$ of total dolichols in the glucose and nitrogen deficient media, respectively. The level of dolichols in yeast cells increased with the age of the cultures. Since both families of dolichols are present in stationary phase cells we postulate that the longer chain dolichols may be responsible for the physico-chemical changes in cellular membranes allowing yeast cells to adapt to nutrient deficient conditions to maintain long-term viability.

Long-chain polyprenols are the products of cis-prenyltransferase which catalyses consecutive additions of isoprene units (in the form of isopentenyl diphosphates) to farnesyl diphosphate. Polyprenyl diphosphate is further dephosphorylated and the $\alpha$-isoprene unit undergoes saturation resulting in dolichol (Chojnacki \& Dallner, 1988; Sagami et al., 1993; Sagami et al., 1996). The chain length of the dolichols is species specific. However, the factor(s) determining the number of isoprene residues is (are) as yet unknown. Dolichols in their phosphorylated form participate in the synthesis of $\mathrm{N}$ - or O-glycosidically linked oligosaccharide chains of glycoproteins and in the formation of glycosylphosphoinositol (GPI) membrane anchors (Herscovics \& Orlean, 1993). No defined role for the free al-

\footnotetext{
This work was partly supported by grant C-2/IX/11 from the French-Polish Centre of Plant Biotechnology and grants 0 182/P04/2002/22 and 3P04A 03722 from the State Committee for Scientific Research (KBN, Poland).

${ }^{\square}$ Corresponding author: Institute of Biochemistry and Biophysics, Polish Academy of Sciences, A. Pawińskiego 5a, 02-106 Warszawa, Poland; fax: (48) 3912 1623; e-mail: babel@ibb.waw.pl
} 
cohol has been established, although this lipid is in the cell and its amount increases with age (Pullarkat \& Reha, 1982). Studies with model membranes indicate that phospholipid fluidity changes in the presence of dolichols (Klosgen \& Helfrich, 1997).

The yeast RER2 gene encoding cis-prenyltransferase that synthesizes polyprenols of 14-18 isoprene residues has been identified (Sato et al., 1999). Mutations in this gene result in disturbances in the correct endoplasmic reticulum (ER) localization of several proteins, and defects in $\mathrm{N}$ - and O-glycosylation. Recently, another yeast gene, SRT1 encoding the cis-prenyltransferase that synthesizes longer chain polyprenols has been cloned (Sato et al., 2001). However, the activity of Srt1p could only be demonstrated in cells, harvested in the stationary phase, lacking Rer2p and transformed with a multicopy plasmid bearing the SRT1 gene. In a paper by Schenk et al. (2001) it was shown that the longer chain dolichols synthesized by the yeast cis-prenyltransferase encoded by overexpressed SRT1 gene with the RER2 gene deleted undergo phosphorylation and the resulting Dol-Ps are used as glycosyl carrier for the assembly of lipid-linked oligosaccharides.

Here we present evidence that the yeast wild type strain W303 displays physiological regulation of the two cis-prenyltransferase encoding genes. Unlike what happens in the early logarithmic phase, stationary phase yeast cells accumulate two polyisoprenoid families, both of them dolichol, of 14-18 and 19-24 isoprene residues. We demonstrate that the induction of the longer chain dolichols synthesis is correlated with a lack of nutrients such as glucose or nitrogen. The possible role of the long chain dolichols is discussed.

\section{MATERIALS AND METHODS}

Strain and culture conditions. The yeast Saccharomyces cerevisiae strain was W303
(Mat a his3-11,15, leu2-3,112, trp1-1, ade2-1, ura3-1, can 1-100 ${ }^{+}$, rho ${ }^{+}$).

Standard complete YPD (1\% yeast extract, $1 \%$ bactopeptone, $2 \%$ glucose) and synthetic complete media were used (Adams et al., 1997). Glucose starvation medium (GS) contained $0.67 \%$ yeast nitrogen base without amino acids, appropriate growth supplements and $0.04 \%$ glucose; nitrogen starvation medium (NS) contained $0.67 \%$ yeast nitrogen base without amino acids and ammonium sulfate, appropriate growth supplements, $2 \%$ glucose and $50 \mu \mathrm{M}$ ammonium sulfate.

For glucose or nitrogen starvation, cells were pregrown in YPD medium, collected at the early logarithmic phase and transferred to GS or NS media, respectively. Cells were incubated in starvation media for $6 \mathrm{~h}$ at $28^{\circ} \mathrm{C}$ with vigorous aeration. Late stationary phase cells were collected after 3 days of growth in YPD medium.

\section{Characterisation of polyprenols synthe-} sized in vivo. Yeast cells were harvested, washed with water and broken with glass beads in $50 \mathrm{mM}$ Tris/HCl buffer, $\mathrm{pH}$ 7.4. After centrifugation at $2000 \mathrm{~g}$ for $10 \mathrm{~min}$ to remove unbroken cells the supernatant was spun at $48000 \boldsymbol{g}$ for $90 \mathrm{~min}$. The pellet was extracted with chloroform/methanol (3:2, v/v). Denatured proteins were discarded by centrifugation. The supernatant containing the extracted lipids was washed three times with $1 / 5$ volume of $10 \mathrm{mM}$ EDTA in $0.9 \% \mathrm{NaCl}$ and evaporated to dryness. The lipids were dissolved in hexane and applied to a silica gel column equilibrated with hexane. A step-wise gradient of 3, 8, 12 and $15 \%$ diethyl ether in hexane led to the recovery of an almost pure fraction of polyprenols eluting in $12 \%$ diethyl ether. Polyprenols were subsequently subjected to HPLC analysis.

The amount of dolichols was estimated by comparison with a quantitative standard of $\mathrm{Dol}_{23}$. The chain length and identity of the dolichols was confirmed by applying mixtures of natural dolichols isolated from pig liver and polyprenols isolated from Ginkgo 
biloba (the isoprenoid standards were obtained from the Collection of Polyprenols, IBB PAS, Warsaw).

Chromatography. Thin-layer chromatography was performed on Silica Gel 60 plates in benzene/ethyl acetate (95:5, v/v). HPLC was performed on an ODS Hypersil $3 \mu \mathrm{m} 60$ $\times 4.5 \mathrm{~mm}$ column at a flow rate of $1.5 \mathrm{ml} / \mathrm{min}$ in a linear gradient program from A: methanol/isopropanol/water (12:8:1, by vol.) to B: hexane/isopropanol (7:3, v/v) in 20 min with the UV detector at $210 \mathrm{~nm}$.

\section{RESULTS}

\section{Analysis of dolichols extracted from the wild-type strain W303 grown on YPD medium}

Polyisoprenoids from yeast cells in the early logarithmic phase $\left(1.3 \times 10^{7}\right.$ cells $\left./ \mathrm{ml}\right)$ grown on YPD medium were extracted. HPLC analysis showed that they are a family of dolichols ranging in size from 14 to 18 isoprene residues (Fig. 1A). Polyisoprenoids extracted
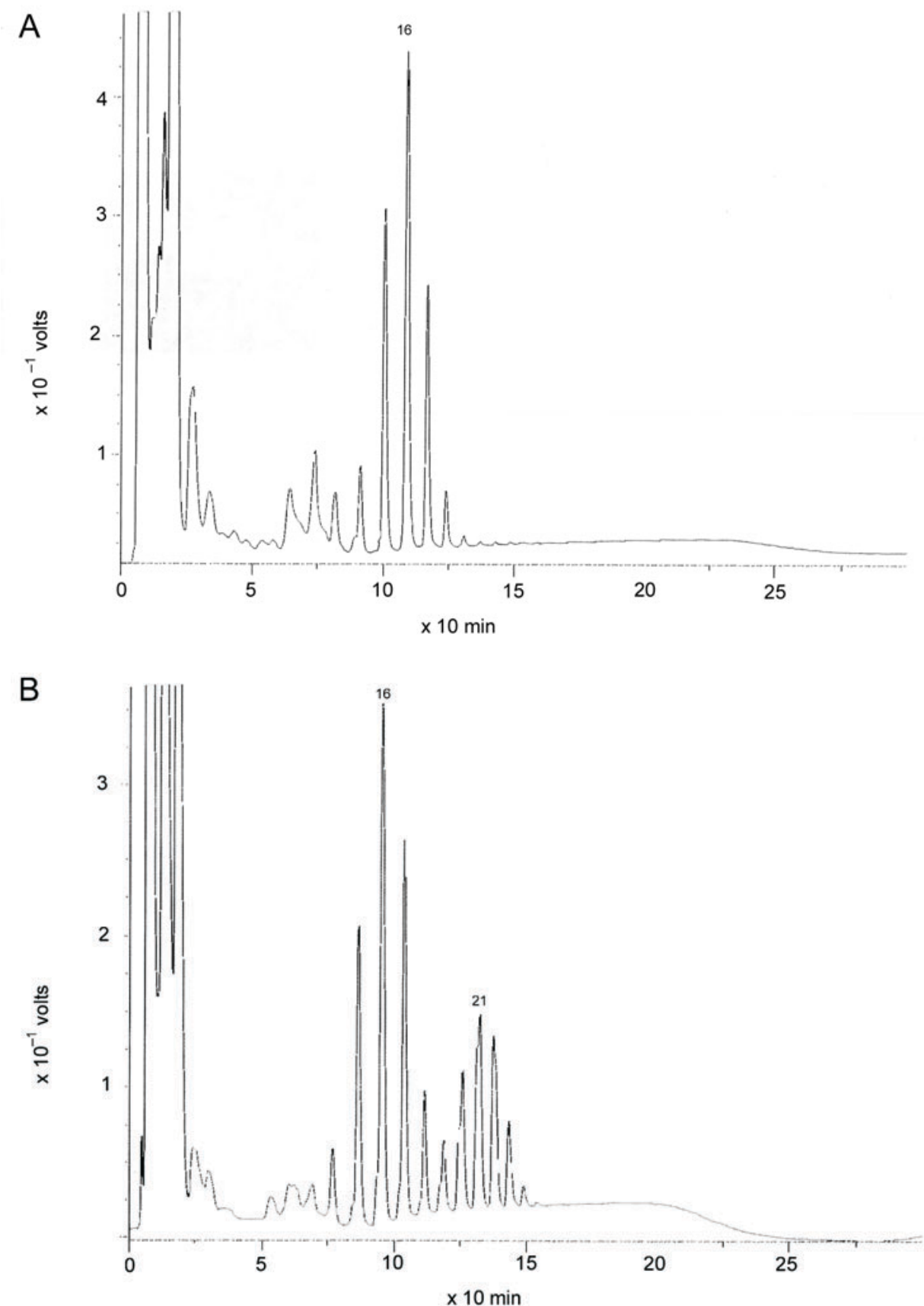

Figure 1. HPLC chromatogram of a purified dolichol fraction prepared from yeast cells cultivated in YPD medium.

A, Early logarithmic phase; B, stationary phase. Numbers above the peaks indicate the dolichols composed of the given number of isoprene residues. Extraction of lipids and HPLC analysis are as described in Materials and Methods. 
from cells in the late stationary phase $(3 \times$ $10^{8}$ cells $/ \mathrm{ml}$ ) displayed a different pattern. There appeared an additional, well defined family of isoprenoids with 19 to 24 isoprene units (Fig. 1B). The nature of this family was determined by cochromatography with a family of polyprenols (15 to 22 isoprene residues) prepared from Ginkgo biloba. The presence of double rather than single peaks (polyprenols elute slightly faster than the corresponding dolichols) indicates that the mixture is composed of dolichols (Fig. 2). The starved cells it represented $10.3 \%$ of total dolichols (Fig. 3).

\section{Accumulation of dolichols with ageing of yeast cultures}

The level of dolichols in yeast cell cultures was monitored. The data presented in Table 1 indicate that yeast cells in the stationary phase accumulate the largest quantity of dolichols. The smallest amount of dolichols was present in yeast in the early logarithmic phase while in

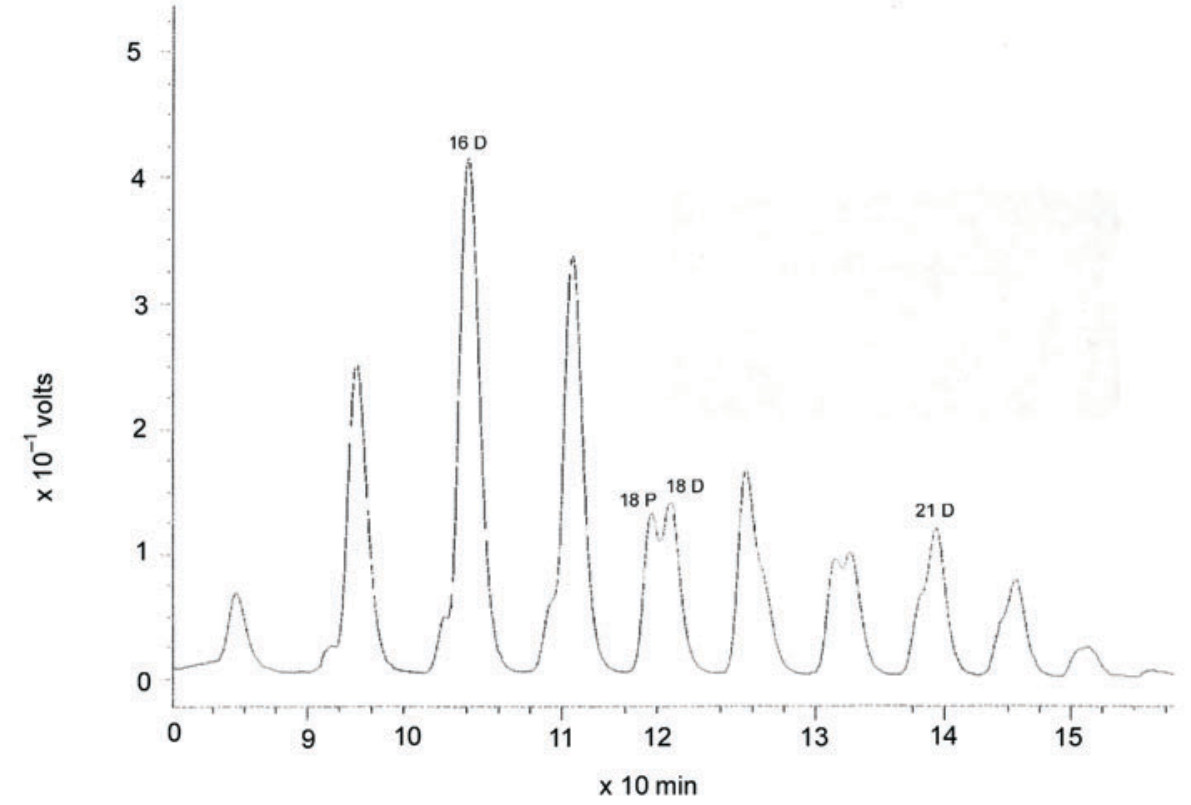

Figure 2. HPLC chromatogram of purified dolichol fraction prepared from yeast cells from stationary phase cochromatographed with a mixture of polyprenols (15-22 isoprene residues) obtained from Ginkgo biloba.

Numbers above the peaks indicate the dolichols composed of the given number of isoprene residues. $\mathrm{P}$, polyprenol; D, dolichol. dolichols in the additional long-chain family represent $39 \%$ of total dolichols (Fig. 3).

\section{Effect of glucose and nitrogen starvation on the synthesis of the long-chain dolichols}

Cells grown in YPD medium to the early logarithmic phase were transferred to a starvation medium lacking glucose or nitrogen and allowed to double their mass. HPLC analyses of prenologues present in both types of starved cells showed the additional family of long-chain dolichols similar to the one observed in cells in the stationary phase (Fig. 4). In glucose starved cells the additional dolichol family represented $13.8 \%$ while in nitrogen cells starved for glucose or nitrogen accumulation was about 2.7 times higher.

\section{DISCUSSION}

The yeast wild-type strain W303 grown in YPD medium synthesizes in the early logarithmic phase dolichols with chain lengths of 14 to 18 isoprene residues and traces of longerchain polyisoprenoids. HPLC chromatography of the lipids extracted from cells in the stationary phase revealed the presence of the typical family of dolichols of 14-18 isoprene residues accompanied by longer chain polyisoprenoid alcohols. According to their chromatographic behaviour these alcohols were 
identified as dolichols, however, further evidence of their structure (mass spectrometry or NMR analysis) is required for a final proof that these alcohols are indeed dolichols.

Contrary to the well documented functions

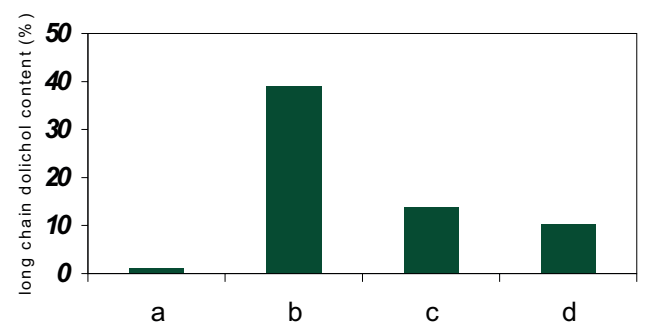

Figure 3. Graphic representation of the percentage of long-chain dolichol content in total dolichol mixtures in yeast cells cultivated in various media.

YPD, (a) early logarithmic phase; (b) stationary phase, and synthetic medium deprived of: (c) glucose; (d) nitrogen. Experimental conditions are as described in Materials and Methods.

of the 14-18 isoprene residue phosphorylated dolichols in protein glycosylation and formation of glycosylphosphoinositol anchors of membrane proteins the role of the additional family of long-chain dolichols (19-24) is not known.

During the logarithmic phase, yeast cells grow primarily by fermentation. In diauxic and postdiauxic phases growth continues but at much lower rate, utilizing energy provided by respiration. Yeast cells enter the stationary phase when depleted of nonfermentable carbon sources as well as when starved for nutrients such as nitrogen, phosphorus or sulfur and possibly other unidentified nutrients. The rapid effects of starvation are obtained by transferring growing cells to a starvation medium lacking a specific required nutrient. The mobilization of endogenous stock nutrients often allows for a limited number of cell divisions during which features of the stationary phase develop (Werner- Washburne et al., 1993). We verified the rapid effect of glucose and nitrogen starvation on the synthesis of dolichols. Cells from the early logarithmic phase growing in YPD medium were shifted to the respective starvation media. The HPLC pattern was similar to that observed for cells in the stationary phase; there were also the additional long-chain dolichols. Quantitative analyses clearly demonstrated that the only difference was in the proportions of the additional family with respect to the constitutive family. In YPD medium, stationary phase long-chain dolichols constituted $39 \%$ of total dolichols while in the glucose or nitrogen starvation media they constituted 13.8 and $10.3 \%$, respectively.

It is noteworthy that we observed an age-associated increase in dolichol levels in yeast cultures. This phenomenon has been reported for mammalian and plant cells (Pullarkat \& Reha, 1982; Paton \& Poulos, 1984; Swiezewska et al., 1994), however, the reason for this is not known. Presented results indicate that the induction of synthesis of the longer chain dolichols correlates with increase in nutrient limitations. We show that both the constitutive and the longer chain dolichols are present in the stationary phase when yeast cells change so as to be able to maintain long-term viability under nutrient deficient conditions.

Table 1. Level of dolichols in yeast cells obtained from cultures grown in different conditions

\begin{tabular}{ll}
\hline Type of culture & $\begin{array}{l}\text { Dolichol content } \\
\mu \mathrm{g} / \mathrm{g} \text { wet mass }\end{array}$ \\
\hline Early logarithmic & $1.01 \pm 0.11$ \\
Glucose deficient & $2.84 \pm 0.22$ \\
Nitrogen deficient & $2.74 \pm 0.28$ \\
Stationary & $4.03 \pm 0.15$ \\
\hline
\end{tabular}

Data are presented as mean \pm S.D. from three independent experiments.

Biological membranes play a central role in the structure and functioning of all cells. Membranes basically define the compartments and determine the nature of communications between the inside and outside of the 

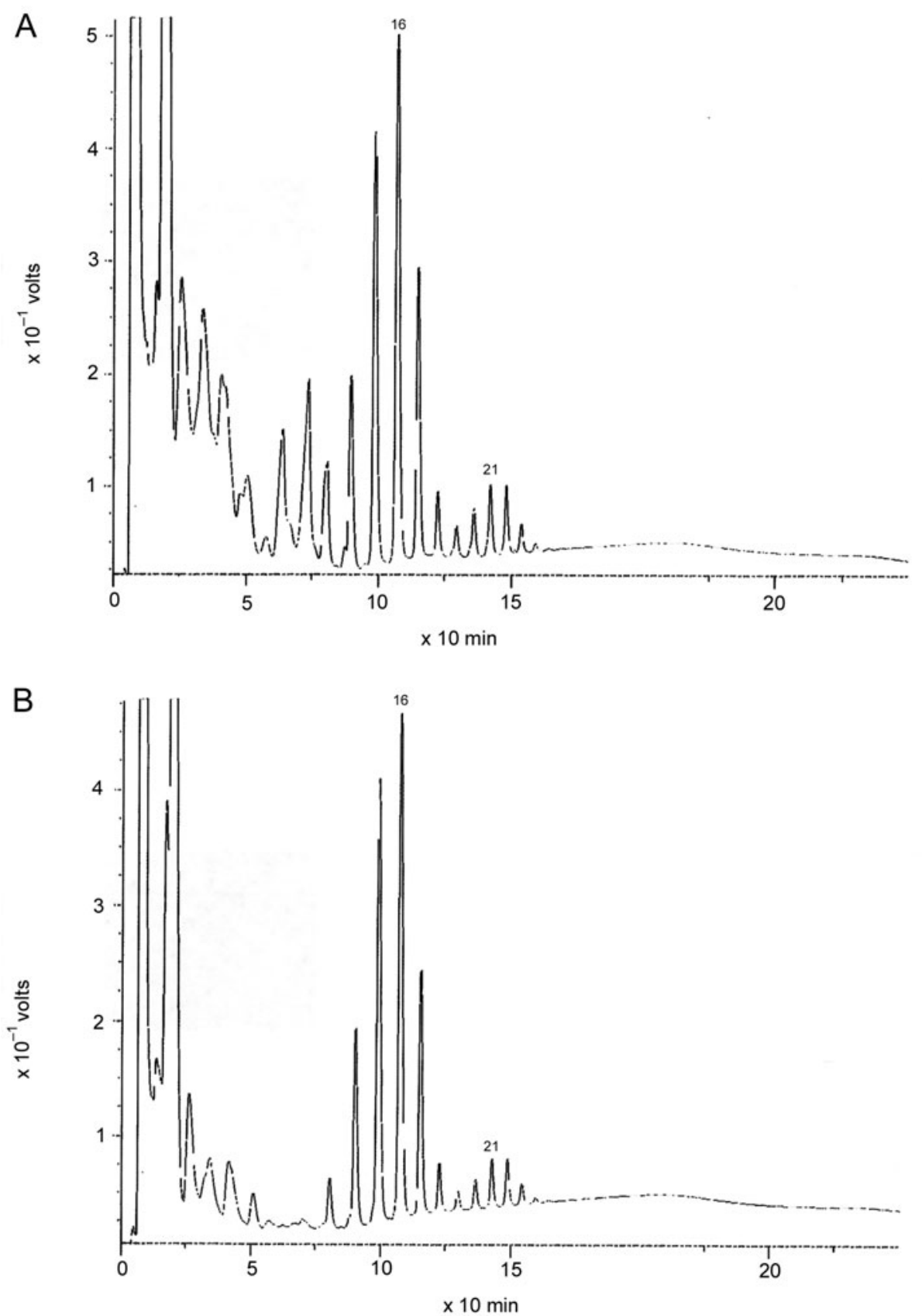

Figure 4. HPLC chromatogram of purified dolichol fraction prepared from yeast cells grown in synthetic media deprived of: A, glucose; B, nitrogen.

Numbers above the peaks indicate the dolichols composed of the given number of isoprene residues. Extraction of lipids and HPLC analysis are as described in Materials and Methods.

cell and subcellular compartments (Gennis, 1989). Experimental evidence indicates that dolichol is sandwiched between the two halves of the phospholipid bilayer (Chojnacki \& Dallner, 1988) and that it exerts a considerable influence on the organization and packing of phospholipids (Valtersson et al., 1985; van Duijn et al., 1986). Very long chain prenols (32 isoprene residues) increase the fluidity and elasticity of lipid membranes and modulate their surface curvature by the formation of fluid microdomains (Janas et al., 2001).
In conclusion, the data reported here document that the appearance of additional longer chain dolichols in stationary phase yeast cells is induced by the lack of major nutrients such as glucose or nitrogen. Dolichols of 14-18 isoprene residues present in the stationary phase secure glycosylation of proteins and proper functioning of the secretory pathway. We postulate that the longer chain dolichols appearing in the stationary phase are possibly responsible for the physico-chemical changes in cellular membranes allowing yeast cells to adapt to nutrient deficient conditions to main- 
tain long-term viability. However, their role in glycosylation of proteins can not be excluded since dolichols with 11 to 32 isoprene residues are phosphorylated and further mannosylated in vitro (Szkopinska et al., 1992).

We are grateful to Dr. Anne-Lise Haenni for critical reading of the manuscript.

\section{R E F E R E N C E S}

Adams A, Gottschling D, Kaiser C, Stearns T. (1997) In Methods in yeast genetics. A Cold Spring Harbor Laboratory Course Manual. CSH Laboratory Press.

Chojnacki T, Dallner G. (1988) The biological role of dolichol. Biochem J.; 251: 1-9.

Gennis RB. (1989) Biomembranes. Molecular structure and function. Springer-Verlag. New York.

Herscovics A, Orlean P. (1993) Glycoprotein biosynthesis in yeast. FASEB J.; 7: 540-50.

Janas T, Walinska K, Chojnacki T, Swiezewska E, Janas T. (2001) Modulation of phospholipid membranes by the long-chain polyprenol $\left(\mathrm{C}_{160}\right)$. Chem Phys Lipids.; 106: 31-40.

Klosgen B, Helfrich W. (1997) Cryo-transmission electron microscopy of a superstructure of fluid dioleoylphosphatidylcholine (DOPC) membranes. Biophys J.; 73: 3016-29.

Paton BC, Poulos AJ. (1984) Dolichol metabolism in cultured skin fibroblasts from patients with "neuronal" ceroid lipofuscinosis (Batten's disease). Inherit Metab Dis.; 7: $112-6$.

Pullarkat R, Reha H. (1982) Accumulation of dolichols in brains of elderly. J Biol Chem.; 257: 5991-3.

Sagami H, Kurisaki A, Ogura K. (1993) Formation of dolichol from dehydrodolichol is catalyzed by NADPH-dependent reductase localized in microsomes of rat liver. $J$ Biol Chem.; 268: 10109-13.

Sagami H, Igarashi Y, Tateyama S. (1996) Enzymatic formation of dehydrodolichal and dolichal, new products related to yeast dolichol biosynthesis. J Biol Chem.; 271: 9560-6.

Sato M, Sato K, Nishikawa S, Hirata A, Kato J, Nakano A. (1999) The yeast RER2 gene, identified by endoplasmic reticulum protein localization mutations, encodes cis-prenyltransferase, a key enzyme in dolichol synthesis. Mol Cel Biol.; 19: 471-83.

Sato M, Fujisaki S, Sato K, Nishimura Y, Nakano A. (2001) Yeast Saccharomyces cerevisiae has two cis-prenyltransferases with different properties and localizations. Implication for their distinct physiological roles in dolichol synthesis. Genes Cells; 6: 495-506.

Schenk B, Rush J, Waechter Ch, Aebi M. (2001) An alternative cis-prenyltransferase activity in yeast that produces polyisoprenols with chain lengths similar to mammalian dolichols. Glycobiology.; 11: 89-98.

Swiezewska E, Sasak W, Mankowski T, Jankowski W, Vogtman T, Krajewska I, Hertel J, Skoczylas E, Chojnacki T. (1994) The search for plant polyprenols. Acta Biochim Polon.; 41: 221-60.

Szkopinska A, Swiezewska E, Chojnacki T. (1992) On the specificity of dolichol kinase and DolPMan synthase towards isoprenoid alcohols of different chain length in rat liver microsomal membrane. Int $J$ Biochem.; 24: 1151-17.

Valtersson C, van Duijn G, Verkleij A, Chojnacki T, de Kruijff B, Dallner G. (1985) The influence of dolichol, dolichol esters, and dolichyl phosphate on phospholipid polymorphism and fluidity in model membranes. $J$ Biol Chem.; 260: 2742-51.

Van Duijn G, Valtersson C, Chojnacki T, Verkleij A, Dallner G, de Kruijff B. (1986) Dolichyl phosphate induces non-bilayer structures, vesicle fusion and transbilayer movement of lipid: a model membrane study. Biochim Biophys Acta.; 861: 211-23.

Werner-Washburne M, Braun E, Johnstone G, Singer R. (1993) Stationary phase in the yeast Saccharomyces cerevisiae. Microbiol Rev.; 57: 383-401. 